\title{
An Effective Policy for Recycling Parts for the Production Management of Consumable Supplies
}

\author{
Satoshi Ito, Tatsuya Komuro, Tomoaki Yamazaki, and Toshiyuki Matsumoto \\ Department of Industrial and System Engineering, \\ Aoyama Gakuin University 5-10-1 Fuchinobe, Sagamihara, \\ Chuo-ku Kanagawa, 252-5258 Japan
}

\begin{abstract}
Recycling has been gaining prominence as a way of preserving natural resources, and the number of companies in the recycling business is increasing. However, there is no effective decision policy for recycling parts. Therefore, this study (1) selects products and models them to simulate the production activities in a recycling company with the help of a computer; (2) designs and analyzes some decision policies for disassembling used products that avoid excessive inventory and limit the use of new materials; and (3) proposes the most effective policy that decides the number of collected products to be disassembled and offsets the deficit with new parts. Finally, the authors proposes balances of the amount of disposes and total cost.
\end{abstract}

Keywords: Recycling, Simulation, Production Management, Inventory.

\section{$1 \quad$ Introduction}

Recently, recycling has been seen as one way of preserving natural resources, and the number of companies in the recycling business has been increasing.

Recycling companies collect and disassemble used products and clean or repair their parts, with which they produce new products. However, most of the collected products are damaged, and the extent of the damage varies among parts. Moreover, the number of recyclable parts that can be acquired from collected products varies (hereafter referred to as the yield rate; the rate of the amount of parts that can be retrieved from a fixed number of products). The difference in yield rates among various parts influences the recycling company greatly. For example, if the yield rate of a certain part is low, the company needs to purchase that part or increase the number of products to be disassembled. If the company intends to recycle natural resources, it should increase the number of products to disassemble in order to limit using new materials. However, having excessive inventory is not desirable, either. For these reasons, the difference in yield rates among the different parts makes it difficult to decide the appropriate number of products to disassemble.

Regarding disassembly operation in recycling, studies on the disassembly-oriented design (Onoda 2003), studies on a optimal ordering and recovery policy for reusable items (KOH,G. 2002), and studies on inventory management of remanufacturable 
products (TOKTAY,B. 2000) are presented. These studies, however, did not consider the difference in yield rates among parts. Moreover, these studies focused on only total cost and did not describe reduction of scraps.

This study, thus, aims to devise a policy regarding the ideal number of products to disassemble that avoids excessive inventory and limits the use of new materials.

\section{$2 \quad$ Target Company and Products}

\subsection{Status of the Target Company and Products}

We selected Plant $\mathrm{N}$, which recycles consumable supplies for copy machines as an Original Equipment Manufacturer (OEM), as the target company. This company has nine processes for recycling products, from collection to shipment. In this study, we focus on the disassembling process.

Target product "AA" is the marking unit of copy machines. It has 31 parts excluding connecting parts such screws or washers. Moreover, it comes in different varieties as shown in Figure 1. "Destination" indicates the client companies to which the products are shipped. "Function" indicates the

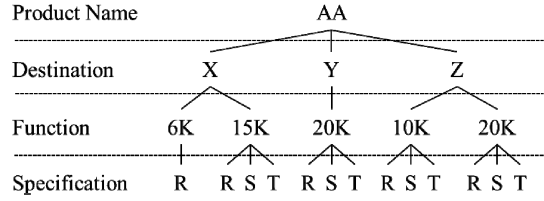

Fig. 1. Product Type capability of printing; for example, " $6 \mathrm{~K}$ " means that the product can print up to 6,000 sheets of paper. There are two methods of preparing parts: one method uses new parts that are bought (hereafter referred to as NewParts) and the other uses recycled parts that are disassembled from collected products (hereafter referred to as ReParts). "Specification" indicates the configuration of NewParts or ReParts. Each R, S, and T specification has its own rule.

\subsection{Current Decision-Making Policy for Disassembling}

In the target process, the current policy regarding the appropriate number of products to be disassembled comes from the required number of the lowest yield rate parts. This policy allows the collection of all the parts from the disassembled products. However, the inventory of parts with a high yield rate becomes larger, which results in a large amount of scraps. Certainly, using the parts of used products instead of new parts and having an excessive inventory of parts are desirable from the environment perspective. However, almost all companies have limited human resources, limited places to store parts and products, and limited funds to utilize scraps in thermal recycles. Thus, to continue the recycling business, the decision-making policy should be concerned with both the environment and economy.

Here, we devise a policy that decides the appropriate number of products to be disassembled, by using computer simulation and considering the above points. 


\section{$3 \quad$ Modeling}

\subsection{Modeling of the Company and the Process}

To analyze the influence of the decision-making policy on production, we simulate the disassembling process of the target company. The model company disassembles collected products and supplies recycled parts to produce new products.

We set the other conditions which is like working time in the model company as per those in the target company, as shown in Table 1. The model process is usually operated by four workers for 6 hours a day.

Table 1. The Conditions in the Model Company

\begin{tabular}{|c|c|c|c|c|}
\hline & $\begin{array}{c}\text { Workers } \\
\text { (people) }\end{array}$ & $\begin{array}{c}\text { Working } \\
\text { Time (hours) }\end{array}$ & $\begin{array}{c}\text { Disassembling } \\
\text { Capability 1 } \\
\text { (/day) }\end{array}$ & $\begin{array}{c}\text { Disassembling } \\
\text { Capability 2 } \\
\text { (/hour) }\end{array}$ \\
\hline regular & 4 & 6 & 180 & 27 \\
\hline Increase 1 & 4 & 7.5 & 200 & 27 \\
\hline Increase 2 & 5 & 7.5 & 240 & 32 \\
\hline
\end{tabular}
Further, to increase production, the number of workers and working hours can be increased to 6 and 7.5 respectively.

Analyzing the amount of collection and shipment in target company, we see that they almost are stable, hence, the amount of collection does not influence whether or not the ReParts are sufficient for production.

\subsection{Modeling of the Product}

A recycling company obtains parts from used products. Therefore, the company's control items for the parts are different from those of general production companies. General production companies mainly control commonality, whether each part adapts to other products or not, and prices. On the other hand, in addition to commonality and price, recycling companies have to control the amount of collection, method of preparing parts, and yield rate of ReParts. As mentioned earlier, the amount of collection does not influence this simulation. Here, we set four control items for the model product: (1) commonality; (2) price of parts; (3) method of preparing parts; and (4) yield rate.

To model the parts, we classify these control items. Then, we categorize the target parts as per these classified control items and regard the corresponding items as model parts.

Before classifying the items, we consider certain factors regarding the control items. The target products have 3 part types: common parts, unique parts, and base parts. The common parts

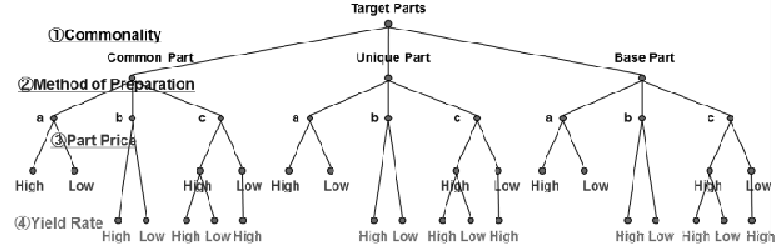

Fig. 2. Classification Items are useful for all product types. On the other hand, the unique parts are useful for each specific product type; however, among the unique parts, some parts can be useful for other product types 
after processing. Hence, these alterable parts (hereafter called base parts) are classified separately. Moreover, the target company does not recycle parts that have a low price and low yield rate; hence, the items that correspond with these conditions are excluded. Considering these factors, we classify the items as shown in Figure 2.

We then categorize the target parts as per the classified control items. We find that the target parts correspond with nine out of the 21 items. Hence, we set these nine items as the model parts. We set the names of the model parts as follows. (Name of model part: $X \mathrm{X}-\mathrm{p} / \mathrm{r}$ )

X: commonality: A: common part; B: unique part; C: base part

x: method of acquisition: a: NewParts(always); b: ReParts(always); c:ReParts(if possible)

p: parts price: Hp: high-price parts; Lp: low-price parts

r: yield rate: Hr: high-yield-rate parts; Lr: low-yield-rate parts

\section{Design of Simulation Conditions}

\subsection{Evaluating Conditions for Designing Decision-Making Policies}

We evaluate the advantages and disadvantages of decision-making policies. We also consider factors such as yield rates, price, commonality, and the number of parts required for production. The following factors are relevant for deciding the number of products to be disassembled:

- Yield rates of parts

It is important to evaluate the part that we regard as the reference value to decide the number of products to be disassembled. If we regard the lowest-yield-rate part as the standard, we will be able to collect each part in sufficient quantity for production but the inventory of some parts will become excessive. On the other hand, if we regard the highest-yield-rate part as the standard, we will collect only the required parts for production. Thus, we select the part numbers required for production as the index to decide whether the part with the highest yield rate or that with the lowest yield rate should be the standard in the disassembling process.

\section{- Prices of parts}

The target parts include both expensive and cheap parts. A recycling company should keep the purchasing costs low by enhancing the percentage of ReParts. Thus, we prioritize the collection of high-priced parts in the disassembling process.

- Commonalities of parts

Unique parts are not compatible with any other type of products. Hence, if the number of unique parts becomes excessive, it will increase the inventory cost. Thus, we prioritize common parts in the disassembling process. 
- Part numbers required for production

Some target parts are acquired only from recycling. Therefore, if a company does not acquire the required number of ReParts, production will stop. Thus, we prioritize ReParts, which cannot be substituted with NewParts.

\subsection{Design of Decision-Making Policies for Disassembling}

By considering the above points, we devise policies for deciding the number of products to be disassembled as follows:

\section{Decision Policy 1: Adjusting to the lowest-yield-rate part}

In this policy, the number of products to be disassembled comes from the required number of lowest-yield-rate parts for production. A key characteristic of this policy is that a company can collect all parts for production from recycling but the volume of inventory will increase.

\section{Decision Policy 2: Adjusting to the highest-yield-rate part}

In this policy, the number of products to be disassembled comes from the required number of highest-yield-rate parts for production. A company can keep the number of disassembled parts and inventory low, but it cannot collect lower-yield-rate parts only from recycling, which makes the purchasing cost high.

\section{Decision Policy 3: Adjusting for the shortfall of NewParts}

In this policy, the number of products to be disassembled comes from the deficit of NewParts. A company acquires a fixed number of NewParts and makes up for the shortfall with ReParts. A characteristic of this policy is that a company can keep the number of disassembled parts lower than that in Decision Policy 1 but the purchasing costs will increase.

We set the upper limit of the fixed number of NewParts at 500 and the lower limit at 100 for simulation considering the capacity of the model company.

\section{Decision Policy 4: Fixing the number of products to be disassembled}

In this policy, the number of products to be disassembled is fixed and the company covers the shortfall with NewParts. A key feature of this policy is that a company can limit the excessive inventory of certain parts but the purchasing costs will increase.

We set the upper limit of the fixed number of products to be disassembled at 500 and the lower limit at 100 for simulation considering the capacity of the model company.

\section{Decision Policy 5: Adjusting to the lowest-inventory part}

In this policy, the number of products to be disassembled comes from the required number of lowest-inventory parts for production. An important feature of this policy is that a company can collect almost all the parts for production but the inventory will increase, although it will be lower than that in decision policy 1.

We set two cases of lowest-inventory parts in this policy: lowest-yield-rate parts and highest-yield-rate parts. 


\section{$5 \quad$ Implementation of Simulation and Analysis of the Results}

\subsection{Input and Output Data}

The input data of the simulation are as follows:

- Company data: disassembling capability, number of workers, and hours of operation in the model company

- Collection and Shipment: actual quantities of collection and shipment of each type of product per day in target company of 454 days

- Parts: commonality, method of acquisition, price, and yield rate

- Initial inventory: the initial inventory of the collected products and parts is 1000 parts.

The output data of the simulation are as follows:

- Operation: unshipped products because of a deficit in parts

- Inventory: the number of collected products before disassembling per day

- Parts: the amount of inventory, purchasing, and scrapping for each part

\subsection{Analysis of the Result in Simulation}

We analyze the simulation results from the viewpoint of unshipped products, the number of products to be disassembled, product inventory before disassembling, inventory of parts, and purchasing amount by using a radar chart. Policy 3 of disassembling includes the cases in which the fixed number of NewParts of 100-500 changes gradually along with the fixed number in every item. Thus, we consider only the upper limit of 500 and the lower limit of 100 in this analysis. Moreover, policy 4 of disassembling includes the cases in which the fixed number of products to be disassembled of 100-500 does not change; thus, we consider only the upper limit of 500 in this analysis.

Figure 3 indicates the influence of each disassembling policy for the model company. It also shows that the number of products to be disassembled is the largest in policy 1 and the lowest in policy 2, while product inventory is inversely proportional to this number. Then, the purchasing number increases in policies 2 and 3 . In policy 2 , the number of products to be disassembled comes from the required number of highest-yield-rate parts; hence, the number of lower-yield-rate parts tends to be low. In policy 3 , the model company's acquisition of a fixed number of NewParts causes the purchasing number to increase. Lastly, all policies except policy 3 (which has a fixed number of NewParts of 500) can keep the scrapping number lower than the current policy can. In the case of the fixed number of NewParts of 500 in policy 3, the purchasing number exceeds the required number of parts for production; thus, the scrapping number increases.

Next, we illustrate the inventory of parts, purchasing number, and scrapping number in Figures 4 and 5 respectively. 


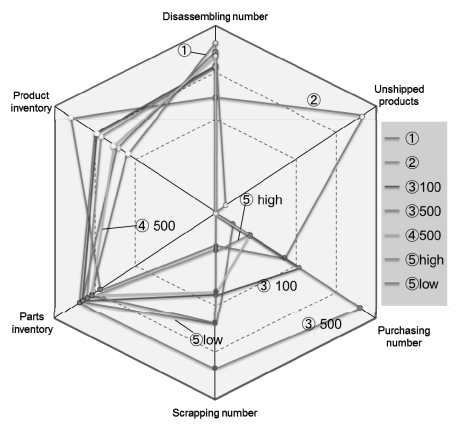

Fig. 3. Influence of disassembling policies

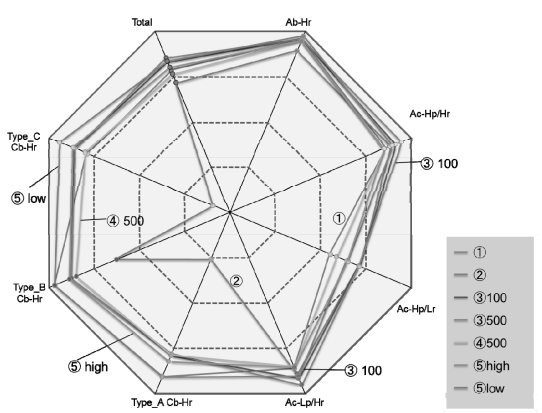

Fig. 4. Parts inventory

1. Changes in the inventory of each part

No major differences in inventory were observed among the policies, except in policy 2, wherein the inventory is kept low but the number of unshipped products becomes high, which we see is the result of inventory shortage. Thus, policy 2 is considered impractical.

In contrast with the current policy (policy 1), policy 4 can keep the inventory low except for Ac-Hp/Lp. The number of products to be disassembled comes from the required number of lowest-yield-rate parts in policy 1; hence, the inventory of Ac$\mathrm{Hp} / \mathrm{Lp}$ is less likely to be excessive.

2. Changes in the purchasing number of each part

From Figure 5, we can see that policies 4 and 5 can keep the purchasing number low, while policy 1 , in which we decide not to buy NewParts, cannot. In policy 1,4 and 5 , NewParts compensate for the deficit of ReParts; hence, the purchasing number tends to be low. On the other hand, in policies 2 and 3, the purchasing number becomes two or five times that in the other policies and is considered very high.

From the above analyses, we think that policy 4 is the most economical and

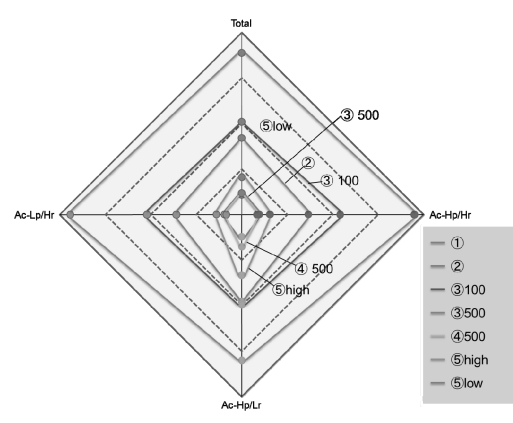

Fig. 5. Purchasing number ecologically sound.

\section{Conclusion}

In this study, we modeled products to simulate the production activities in a recycling company with the help of a computer. Second, we designed and analyzed some decision policies for disassembling used products that avoid excessive inventory and limit the use of new materials. Finally, we devised the most effective policy that 
decides the number of collected products to be disassembled and offsets the deficit with new parts from the result of simulation.

This study considered only one process in the target company; for future studies, we need to extend the target size to the whole factory and make the production flow efficient using simulation.

Acknowledgement. This study is funded by Grant-in-Aid for Scientific Researches (numbered25350262). The authors would like to express their gratitude to the organization.

\section{References}

1. Onoda, H., Nagata, K., Notomi, M.: A Study on the Disassembly-Oriented Design. Design Engineering 38(12), 648-654 (2003)

2. Koh, G., Hwang, H., Sohn, I., Ko, S.: An optimal ordering and recovery policy for reusable items. Computer Industrial Engineering 43(1/2), 59-73 (2002)

3. Toktay, B., Wein, M., Zenios, A.: Inventory Management of Remanufacturable Products. Manag. Sci. 46(11), 1412-1426 (2000) 